\title{
Variabilidade dos ciclos anual e interanual da radiação de ondas longas emergentes sobre a América do Sul e vizinhanças
}

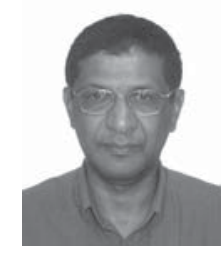

Nelson J. Ferreira ${ }^{1} \&$ Helen da C. Gurgel ${ }^{2}$

\author{
1 Divisão de Sensoriamento Remoto/INPE. CP 515, CEP 12201-970, São José dos Campos, SP. Fone: (12) 3945-6643. \\ E-mail: nelson@ltid.inpe.br (Foto) \\ 2 Divisão de Sensoriamento Remoto/INPE. E-mail: helen.gurgel@ig.com.br
}

Protocolo $60-7 / 5 / 2001$

\begin{abstract}
Resumo: Este trabalho analisa a variabilidade anual e interanual da radiação de ondas longas emergentes (ROL) derivadas do sensor "Advanced Very High Resolution Radiometer" (AVHRR) sobre a América do Sul e vizinhanças. As análises foram feitas utilizando-se médias mensais de ROL para o período de janeiro de 1982 a dezembro de 1993 e a técnica de estatística multivariada de análise por componentes principais (ACP). Os resultados obtidos possibilitaram a caracterização espacial dos padrões dos principais sistemas de tempo que atuam sobre o Brasil. Em relação à $\mathrm{ACP}$, observou-se que a primeira componente representa o padrão médio da atividade convectiva; a segunda e quarta componentes estão associadas, respectivamente, aos modos verão/inverno e primavera/outono do ciclo anual; a terceira componente mostra as variações moduladas por eventos de El Niño, e a quinta componente está associada ao ciclo semi-anual.
\end{abstract}

Palavras-chave: atividade convectiva, componentes principais, clima

\section{Annual and interannual variability of outgoing longwave radiation over South America and neighboring regions}

\begin{abstract}
A principal component analysis was used to characterize the annual and interannual variability of outgoing longwave radiation over South America and neighboring regions. The data sample is comprised of monthly Advanced Very High Resolution Radiometer (AVHRR) data, covering the period January 1982 - December 1993. The results obtained characterize the behavior of the spatial pattern of the dominant weather systems acting over Brazil. The first component represents the mean convective activity pattern; the second and the fourth ones are respectively associated with the summer/winter and spring/autumn modes of the annual cycle. The third component shows spatial variability modulated by El Niño and the fifth one is associated with the semi-annual cycle.
\end{abstract}

Key words: convective activity, principal components, climate

\section{INTRODUÇÃO}

Uma variável muito utilizada para a caracterização climática nos trópicos é a emissão para o espaço de radiação de ondas longas (ROL) medida por satélites. Essa variável está relacionada à lei de Stefan-Boltzmann aplicada à temperatura da superfície terrestre, de acordo com a qual, quanto menor a temperatura da superfície, menor será a emissão de ondas longas. Em meteorologia, a ROL é muito utilizada para indicar a presença de nuvens convectivas nos trópicos, cujos topos atingem altitudes elevadas e, normalmente, estão associadas à ocorrência de precipitação. Quanto mais alto estiver o topo de uma nuvem, mais baixa será a emissão de ondas longas, e por isso a ROL pode ser utilizada como indicador de precipitação, porém, essa variável se baseia na temperatura da superfície dos alvos, devendo ser utilizada apenas nas regiões tropicais, pois a baixa temperatura da superfície terrestre das latitudes médias e altas, principalmente no inverno, propicia a interpretação errônea da presença de nuvens nessas regiões (Ohring et al., 1984).

Diversos estudos já exploraram o uso de ROL para caracterização climática. Um dos trabalhos pioneiros foi realizado por Heddinghaus \& Krueger (1981) que utilizaram médias mensais de ROL para caracterizar as variações anuais e interanuais nos trópicos, através da técnica estatística de Funções Ortogonais Empíricas ("Empirical Orthogonal Function" - EOF) que também é conhecida como Análise por Componentes Principais (ACP). Eles constataram que a primeira componente representava o modo verão/inverno do ciclo anual; a segunda descrevia o modo de primavera/outono e a terceira o ciclo semi-anual. A partir de dados de anomalia de ROL, Kousky \& Kayano (1994) diagnosticaram variações, tanto no padrão da convecção 
tropical, como na intensidade do vento zonal na alta troposfera tropical. Essas variações foram associadas a padrões atmosféricos vinculados a situações de extremo da oscilação do sul (OS) na América do Sul.

Em estudo também sobre série histórica de ROL, Kayano et al. (1995) analisaram o impacto de se corrigirem ou não os erros ocasionados pelos diferentes horários em que o satélite cruza o equador. Através da técnica EOF eles mostraram que a utilização da série não corrigida de ROL induzia a obtenção de uma componente (segunda) sem significado físico, porém, ao analisarem uma série histórica de anomalia de ROL (1974 a 1996), Waliser \& Zhou (1997) constataram que as três primeiras componentes estão associadas às variações interanuais. A primeira componente destacou os eventos de El Niño de 1975$76,1982-1983,1987,1988-89$ e 1992, a segunda os eventos de 1972-73 e 1986-87 e a terceira o evento de 1982-83. A quarta componente revelou as variações de ROL ocasionadas por mudança no horário da passagem do satélite.

Andreoli (1998), utilizando pêntadas (média de cinco dias) de ROL e EOF, constatou que as máximas variâncias interanuais e totais nos trópicos estão associadas às variações sazonais da convecção tropical. Ela mostrou, também, que no verão as correlações negativas/positivas de ROL nos trópicos estão associadas a regiões de intensa/reduzida convecção. Estudos específicos envolvendo análises dos ciclos anual e interanual de ROL nos trópicos foram documentados por vários autores, como Heddinghaus \& Krueger (1981), Aceituno (1988), Horel et al. (1989) e Kayano et al. (1995). Entretanto, esses trabalhos não enfatizaram os aspectos climáticos a nível regional. Os resultados das análises de ROL apresentados a seguir têm como objetivo auxiliar a identificação dos padrões climáticos associados à ROL na América do Sul e vizinhanças.

\section{MATERIAL E MÉTODOS}

Os dados de ROL utilizados neste trabalho fazem parte dos conjuntos de dados meteorológicos disponibilizados pelo "National Centers for Environmental Prediction" (NCEP) e foram fornecidos pelo NOAA-CIRES Climate Diagnostic Center, Boulder, Colorado, através da homepage http://www.cdc.noaa.gov/.

Esses dados são médias mensais em valores reais de 32 bits, organizados em pontos de grade com resolução espacial de $2,5 \times 2,5^{\circ}$ de latitude/longitude, e cobrem todo o globo terrestre para o período de janeiro de 1979 a dezembro de 1995; porém, o período utilizado foi de janeiro de 1982 a dezembro de 1993. Detalhes sobre esses dados encontram-se em Kalnay et al. (1996).

O cálculo do ROL é feito a partir de medições radiométricas obtidas pelo sensor AVHRR/NOAA na faixa espectral entre 10,5 a $12,5 \mu \mathrm{m}$ (Waliser \& Zhou, 1997). O procedimento para obtenção e processamento dessa variável foi documentado por Gruber \& Krueger (1984), Ohring et al. (1984) e Janowiak et al. (1985). Os dados de ROL utilizados em unidades de $\mathrm{W} \mathrm{m}^{-2}$ foram selecionados exclusivamente pelos satélites com passagem à tarde, cujo valor é obtido através da média do fluxo de ROL em uma janela de 2,5 x 2,5 $5^{\circ}$ de latitude/longitude (Kalnay et al., 1996). A visualização e a manipulação dos dados foram feitas utilizando-se a rotina "Grid Analysis and Display System" (GRADS), fornecida pelo "Center for Ocean-Land-
Atmosphere-Interactions" (COLA); informações completas sobre esta rotina podem ser encontradas em Doty et al. (1997).

A ACP utilizada nesse estudo é uma técnica de estatística multivariada muito útil na investigação de relações existentes entre um conjunto de variáveis correlacionadas. Através da ACP, pode-se transformar um conjunto de dados, inicialmente correlacionados em um novo conjunto de variáveis não correlacionadas. $\mathrm{O}$ novo conjunto é obtido através de combinações lineares das variáveis originais, de tal forma que a maior parte das informações se concentra nas primeiras componentes. O cálculo da ACP envolve, essencialmente, as seguintes etapas (Haan, 1977; Richards, 1986 e Wilks, 1995): obtenção da matriz de variância-covariância ou correlação; cálculo dos autovalores e autovetores; transformação linear dos conjuntos de dados e obtenção dos "loadings" (correlação entre os dados originais e as componentes).

\section{RESULTADOS E DISCUSSÃO}

Tem-se, a seguir, uma descrição do comportamento médio de ROL sobre a América do Sul e vizinhanças, durante o período de estudo. Embora as características dessa variável já tenham sido documentadas em outros estudos, esta discussão serve de base para o entendimento do comportamento dos padrões espaciais das componentes principais de ROL, descritas posteriormente.

\section{Médias de ROL}

Através das médias mensais para cada ano do período de estudo (1982-1993) obtiveram-se as médias sazonais de ROL. Observando-se a Figura 1, é possível se constatar que na primavera e no verão os valores mais baixos de ROL, associados à atividade convectiva, cobrem grande parte do território brasileiro. Esta característica ocorre devido à presença dos diversos sistemas de tempo que atuam em nosso continente. Na região equatorial, a faixa com valores baixos de ROL está associada com a Zona de Convergência Intertropical (ZCIT), que é um dos principais sistemas de tempo nos trópicos. Devido à sua estrutura dinâmica, a ZCIT tem papel decisivo na caracterização das diferentes condições de tempo e de clima, em diversas áreas desta região (Ferreira, 1996). Tal zona contribui para o transporte de umidade do Oceano Atlântico para o continente, aumentando a precipitação em parte da região norte e nordeste do país; detalhes sobre este mecanismo podem ser encontrados em Uvo (1989).

Observa-se, também, na primavera e no verão (Fig. 1) uma banda de ROL, com valores relativamente baixos e orientação NW/SE, estendendo-se do sul da região Amazônica até o Oceano Atlântico Sudoeste. Esta banda de nebulosidade é denominada Zona de Convergência do Atlântico Sul (ZCAS), além de ser um dos mais importantes fenômenos de tempo na escala intrasazonal, que ocorre durante o verão na América do Sul. A ZCAS é a responsável por episódios de enchentes que atingem diversas regiões do País, tais como a Sul e a Sudeste; além disso, ela está associada com as frentes frias que se formam no sul do continente que, ao se encontrarem com o ar tropical no sudeste e centro-oeste, estacionam aumentando a chuva durante o verão (Kodama, 1993; Rocha \& Gandu, 1996). Observa-se, também, na região amazônica, valores de ROL 
A. Primavera

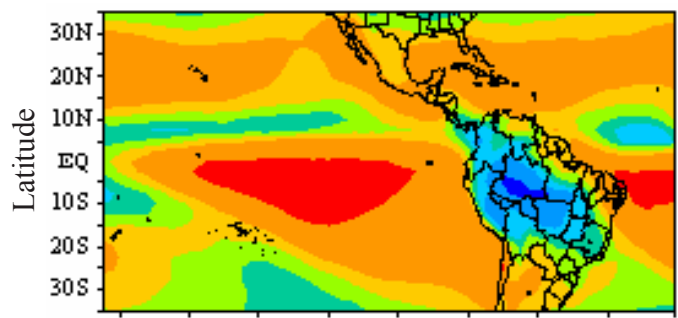

B. Verã̃o

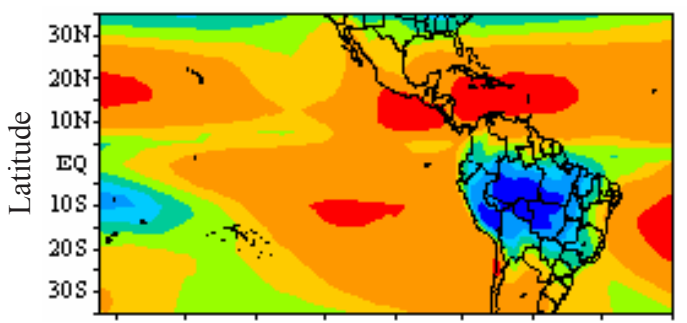

C. Outono

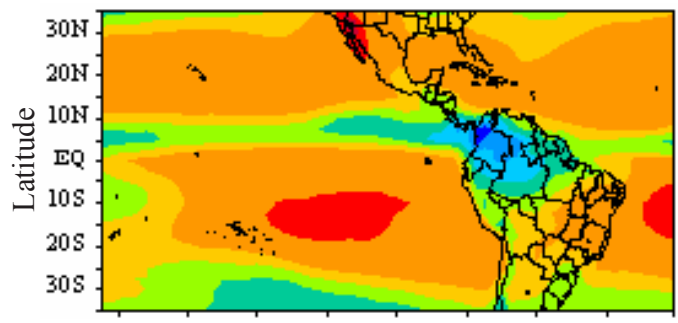

D. Inverno

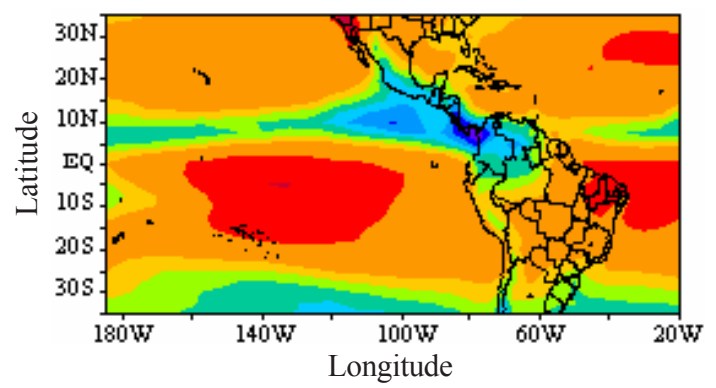

Figura 1. Média sazonal (primavera, verão, outono e inverno) de ROL, em W m², para o período de 1982 a 1993

bastante baixos e abrangentes. Neste caso, a convecção está associada à circulação anticiclônica de grande escala na alta troposfera chamada Alta da Bolívia (Virji, 1981). No outono e no inverno, a atividade convectiva diminui e os valores de ROL sobre o Brasil aumentam consideravelmente, em virtude da estabilidade atmosférica. Nesta época, a ZCIT se desloca para o Hemisfério Norte, seguindo o movimento do Sol, e a ZCAS desaparece. Constata-se, ainda, que a faixa com valores relativamente baixos (210 a $230 \mathrm{~W} \mathrm{~m}^{-2}$ ) de ROL no sul do continente, se desloca para o norte, atingindo a região sul do Brasil no seu máximo, em razão da queda da temperatura da superfície terrestre nesta época do ano.

Análise por componentes principais: A interpretação dos resultados da ACP padronizada, realizada no conjunto de dados de ROL, se baseia na análise das imagens componentes e nos gráficos de autovetores. O gráfico de autovetores, que é uma série temporal, ilustra o peso de cada uma das 144 imagens na obtenção de cada imagem componente (coeficientes de cada componente). Ressalta-se que, quando o autovetor é negativo, é indício de que este mês tem padrão inverso do demonstrado na imagem componente, ou seja, se o valor de ROL está aparecendo alto na imagem componente, na realidade, naquele mês ele foi baixo.

Para realçar a visualização dos dados, aplicou-se um realce linear nas imagens componentes, efetuado de maneira que os dados ficassem com distribuição simétrica em torno do valor zero. Dessa forma, é possível verificar a intensidade e a ocorrência dos valores positivos e negativos, tal que os valores positivos aumentem gradualmente do verde ao vermelho, e os negativos do azul ao preto. Esses valores podem ser considerados anomalias positivas ou negativas, em relação à média mensal de ROL. No caso do ROL, quando ocorre anomalia positiva há aumento dessa variável, isto é, diminuição

A.
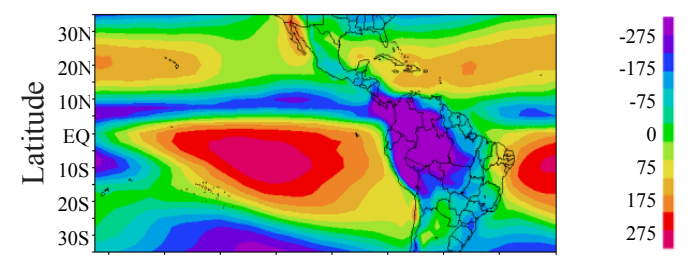

B.
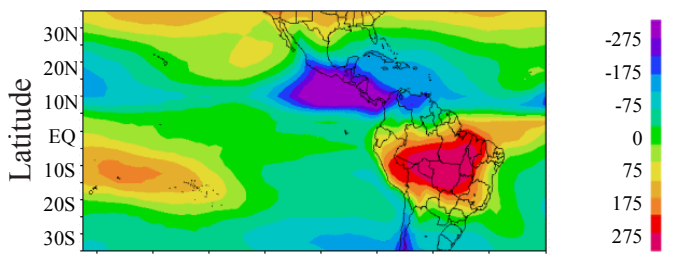

C.
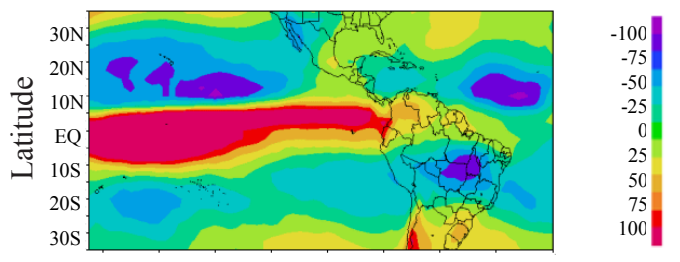

D.
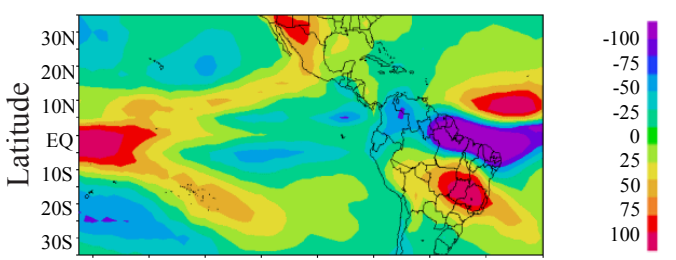

E.
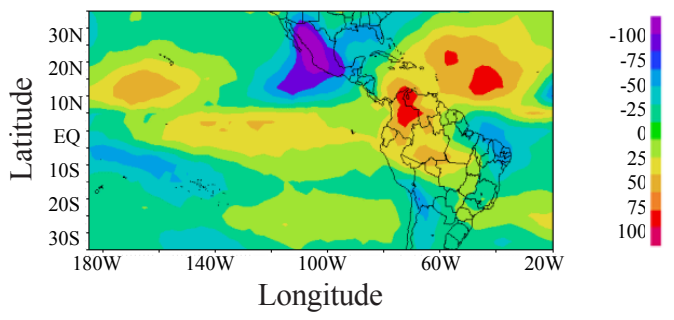

Figura 2. Padrões da análise por componentes principais derivados de dados mensais de ROL, período de 1982-1993: (A) primeiro modo; (B) segundo modo; (C) terceiro modo; (D) quarto modo; (E) quinto modo 
de nuvens convectivas e, quando se dá anomalia negativa, existe diminuição do ROL e, conseqüentemente, aumento da atividade convectiva. Quanto mais fortes forem as anomalias, maior será a relação da região anômala com a série temporal.

Análise da primeira componente: A primeira componente do conjunto de dados de ROL mostra o comportamento médio dessa variável durante o período considerado. Na Figura 2A, observa-se a presença de subsidência (tonalidade vermelha) nos Oceanos Pacífico e Atlântico, associada a altos valores de ROL. A ZCIT localiza-se em torno de $6^{\circ} \mathrm{N}$, na vizinhança do equador meteorológico. Sobre a América do Sul, a atividade convectiva mais acentuada localiza-se sobre a Amazônia, e a de menor sobre a região Nordeste do Brasil. A faixa de nebulosidade, que se estende para o sudeste a partir da Amazônia, reflete a ZCAS ou a posição média das frentes frias. Os autovetores desta componente (Fig. 3A) indicam que em anos de El Niño acentuado $(1983,1992)$ ocorrem variações no campo mensal de ROL, principalmente no início do ano. Esta componente explica $46,47 \%$ da variância total.

Análise da segunda componente: A segunda componente de ROL (Fig. 2B) define muito bem o ciclo anual, conforme mostrado no gráfico dos autovetores (Fig. 3B) e representa
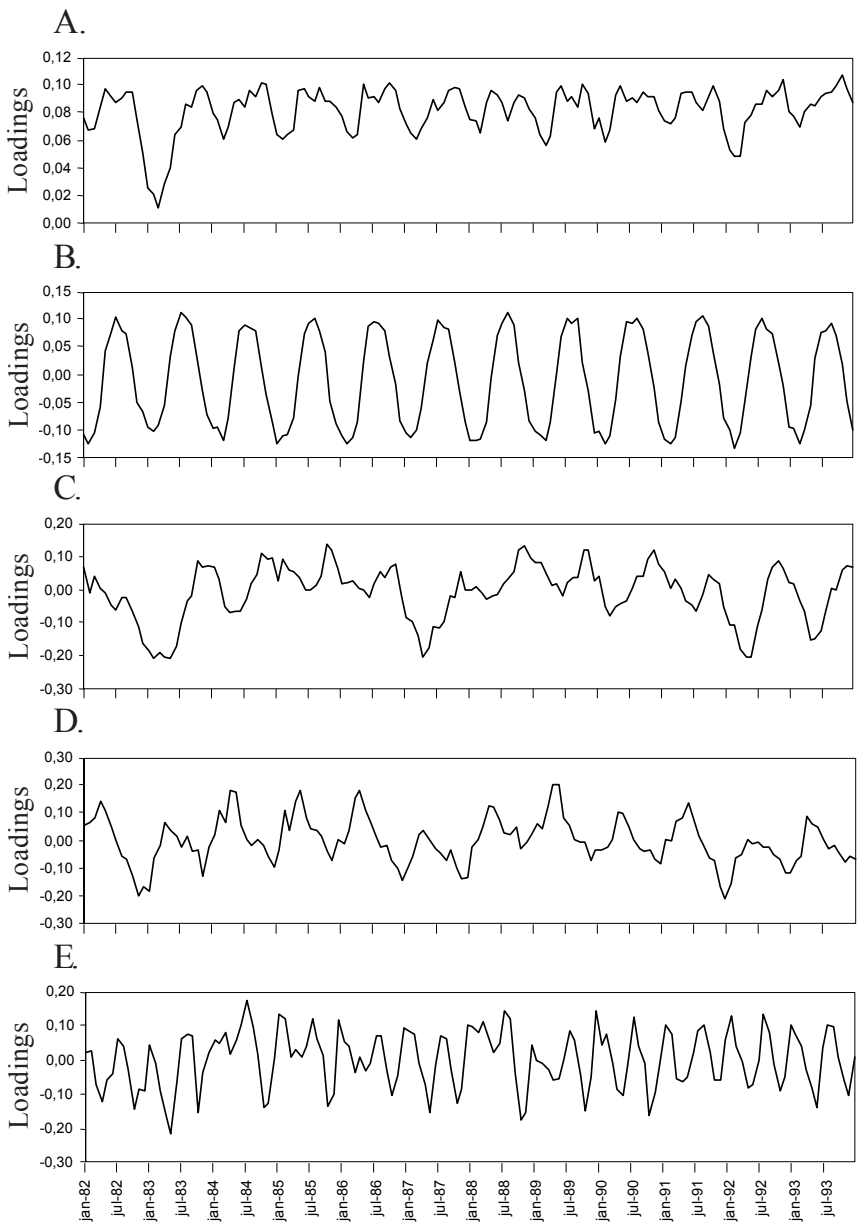

Figura 3. Série temporal dos autovetores/loadings (eixo Y) das componentes de ROL: (A) primeira componente; (B) segunda componente; (C) terceira componente; (D) quarta componente; (E) quinta componente
$21,40 \%$ da variância total. Em função do domínio interhemisférico (hemisférios Norte e Sul) utilizado, essa componente mostra o padrão de verão de cada hemisfério. Com auxílio dessas figuras, observa-se que:

- durante o verão do Hemisfério Sul, a atividade convectiva é bastante acentuada sobre a Amazônia brasileira, tendo o seu máximo no mês de fevereiro; nela, a atividade convectiva interage com duas faixas de nebulosidade, a ZCAS, que se estende para o Atlântico Sudoeste, e a ZCIT, ao longo do equador no Oceano Atlântico. Observa-se também que a ZCAS é mais ativa sobre o continente;

- no verão do Hemisfério Norte a atividade convectiva acentuada se localiza sobre a América Central e tem seu máximo em julho, época em que a ZCIT se situa em torno de $8^{\circ} \mathrm{N}$, sofrendo pequenas descontinuidades, sendo mais ativa na América Central. No verão do Hemisfério Sul, a ZCIT se localiza próximo ao equador, estando inativa principalmente no Oceano Pacífico Equatorial Leste. A nebulosidade sobre o Pacífico Tropical Oeste está associada à Zona de Convergência do Pacífico Sul (ZCPS). A segunda componente revela que o modo verão é bem definido, sofrendo pouca variabilidade interanual e apresentando maior magnitude no verão austral.

Análise da terceira componente: A terceira componente (Figs. 2C e 3C) reflete situações de primavera/outono, porém associadas ao fenômeno El Niño. Sobre o Oceano Pacífico Central (linha do equador) ocorre uma faixa convectiva bastante ativa, enquanto na América do Sul se encontra baixa atividade convectiva sobre o Brasil central e alta sobre o sul do continente, evidenciando a queda da precipitação que ocorre no norte e nordeste do Brasil, e o aumento da precipitação no sul do País, quando há ocorrência do El Niño, conforme documentado por CPTEC (1998). Neste caso, o sinal da atividade convectiva no Oceano Pacífico Central associado à variabilidade interanual, é maior que o ciclo anual (primavera/ outono). Essa componente representa 9,76\% da variância total.

Análise da quarta componente: A quarta componente (Figs. 2D e 4D) revela o padrão primavera/outono do ciclo anual e representa 3,69\% da variância total. No Hemisfério Sul observase, entre setembro e dezembro, uma faixa de nebulosidade que se estende do sul da Amazônia até o Oceano Atlântico Sudoeste. Esta faixa de nebulosidade está associada a incursões equatoriais de frentes frias (Kousky, 1979) que, nesta época do ano, chegam ao sul da região Nordeste, contribuindo para o regime de chuva. Nesta época, a ZCIT está localizada em torno de $8^{\circ} \mathrm{N}$ no Atlântico Norte e ao longo do equador no Oceano Pacífico Oeste. Ao contrário do observado no modo de verão, a ZCIT sofre descontinuidades no Hemisfério Norte; observa-se, também, que a ZCPS se estende para o Pacífico Sudeste, nas proximidades de $120^{\circ} \mathrm{W}$. Por outro lado, entre abril e maio, a costa norte do Brasil é bastante influenciada pela precipitação associada à atividade convectiva ao longo da ZCIT, enquanto as regiões sul e sudeste se tornam relativamente secas. Comparando-se com o modo verão, constata-se que o modo primavera/outono sofre variabilidade interanual acentuada, principalmente em eventos de El Niño. Na primavera austral, a magnitude dessa variabilidade é maior que na primavera boreal. 
Análise da quinta componente: A quinta componente (Figs. $2 \mathrm{E}$ e $3 \mathrm{E}$ ) está associada ao ciclo semi-anual. Ela não apresenta significado físico evidente, como no caso das outras componentes. Os picos positivos da série temporal ocorrem em dezembro/janeiro e julho e os negativos em abril/maio e outubro. A variabilidade interanual do ciclo semi-anual é relativamente regular, sofrendo pequenas alterações em anos de El Niño. Essa componente representa $2,15 \%$ da variância total. Nenhuma das demais componentes apresentou ciclos perceptíveis nem significados físicos evidentes.

\section{CONCLUSÕES}

1. A primeira componente está essencialmente associada ao padrão médio da atividade convectiva.

2. As segunda e quarta componentes estão associadas aos modos verão/inverno e primavera/outono do ciclo anual.

3. O padrão temporal da terceira componente é modulado por eventos de El Niño durante o outono austral.

4. A quinta componente está associada ao ciclo semi-anual.

\section{AGRADECIMENTOS}

Os autores agradecem aos revisores anônimos que contribuíram significativamente para o aprimoramento deste trabalho; ao Conselho Nacional de Desenvolvimento Científico e Tecnológico, Processo Número 300486/96-0 pelo suporte, e a María Silvia Pardi Lacruz e Claudia Cristina dos Santos, pela ajuda na edição das figuras.

\section{LITERATURA CITADA}

Aceituno, P. On the functioning of the Southern Oscillation in the South American sector. Part 1: surface climate. Monthly Weather Review, Boston, v.116, n.3, p.505-524, 1988.

Andreoli, R.V. Variabilidade interanual de campos atmosféricos totais e zonalmente assimétricos. São José dos Campos: INPE-6776-TDI/637, 1998. 95p. Dissertação Mestrado

CPTEC - Centro de Previsão de Tempo e Estudos Climáticos. Conseqüências do fenômeno El Niño sobre o território brasileiro e perspectivas para 1998. http://www.cptec.inpe.br/ products/elninho. Jan. 1998.

Doty, B.E.; Kinter III, J.L.; Fiorino, M.; Hooper, D.; Budich R.; Winger K.; Schulzweida, U.; Calori, L.; Holt, T.; K. Meier, K. The grid analysis and display system (GrADS): An update for 1997. In: International Conference on IIPS for Meteorology, Oceanography and Hydrology, 13, 1997, Longbeach. Proceedings... Longbeach: American Meteorological Society, 1997, p.117.

Ferreira, N.S. Zona de convergência intertropical. Climanálise [on line]. n. Especial, Out. 1996. <http://www.cptec.inpe.br/ products/climanalise $>$. Dez. 1999.

Gruber, A.; Krueger, A.F. The status of the NOAA outgoing longwave radiation data set. Bulletin of the American Meteorological Society, Boston, v.65, n.9, p.958-962, 1984.

Haan, C.T. Statistical methods in hydrology. Ames: Iowa State University Press, 1977. 378p.
Heddinghaus, T.R.; Krueger, A.F. Annual and interannual variations in outgoing longwave radiation over the tropics. Monthly Weather Review, Boston, v.109, n.6, p.1208-1218, 1981.

Horel, J.D.; Hadmann, A.N.; Geisler, J.E. An investigation of the annual cycle of convective activity over the tropical Americas. Journal of Climate, Boston, v.2, n.11, p.1388-1403, 1989.

Janowiak, J.E.; Krueger, A.F.; Arking, P. A. Atlas of outgoing longwave radiation derived from NOAA satellite data. Washington, DC: National Oceanic and Atmospheric Administration, Silver Spring, Md., 1985. NOAA Atlas 6, 44p.

Kalnay, E.; Kanamitsu, M.; Kistler, R.; Collins, W.; Deaven, D.; Gandin, L.; Iredell, M.; Saha, S.; White, G.; Woollen, J.; Zhu, Y.; Chelliah, M.; Ebisuzaki, W.; Higgins, W.; Janowiak, J.; Mo, K. C.; Ropelewski, C.; Wang, J.; Leetmaa, A.; Reynolds, R.; Jenne, R.; Joseph, D. The NCEP/NCAR 40-year reanalysis project. Bulletin of the American Meteorological Society, Boston, v.77, n.3, p.437-471, 1996.

Kayano, M.T.; Kousky, V.E.; Janowiak, J.E. Outgoing longwave radiation biases and their impacts on empirical orthogonal function modes of interannual variability in the tropics. Journal of Geophysical Research, New Brunswick, v.100, n.D2, p.3173-3180, 1995.

Kodama, Y.M. Large-scale common features of subtropical convergence zones (the Baiu frontal zone, the SPCZ, and the SACZ) Part I: Characteristics of subtropical frontal zones. Journal of the Meteorological Society of Japan, Tsukuba, v.71, n.7, p.581-610, 1993.

Kousky, V.E. Frontal influences on Northeast Brazil. Monthly Weather Review, Boston, v 107, n.9, p.1140-1153, 1979.

Kousky, V.E.; Kayano, M.T. Principal modes of outgoing longwave radiation and $250 \mathrm{mb}$ circulation for the South American sector. Journal of Climate, Boston, v.7, n.7, p.1131-1143, 1994.

Ohring, G.; Gruber, A.; Ellingson, R. Satellite determination of the relationship between total longwave radiation flux and infrared window radiance. Journal of Climate and Applied Meteorology, Boston, v.23, n.3, p.416-425, 1984.

Richards, J.A. Remote sensing digital images analysis: An introduction. Berlin: Springer-Verlag, 1986. 281p.

Rocha, A.M.G.C; Gandu, A.W. A zona de convergência do Atlântico Sul; Climanálise Especial, http://www.cptec.inpe.br/ products/climanálise/. Out. 1996.

Uvo, C.B. A zona de convergência intertropical (ZCIT) e sua relação com a precipitação da Região Norte do Nordeste Brasileiro. São José dos Campos: INPE-4887-TDI/378, 1989, 99p. Dissertação Mestrado

Virji, H. A preliminary-study of summertime tropospheric circulation patterns over South America estimated from cloud winds. Monthly Weather Review, Boston, v.119, n.3, p.599-610, 1981.

Waliser, D.E; Zhou, W. F. Removing satellite equatorial crossing time biases from the OLR and HRC databases. Journal of Climate, Boston, v.10, n.9, p.2125-2146, 1997.

Wilks, D.S. Statistical methods in the atmospheric sciences: An introduction. San Diego: Academic Press, 1995. 467p. 